\title{
The effect of telomerase activity on vascular smooth muscle cell proliferation in type 2 diabetes in vivo and in vitro
}

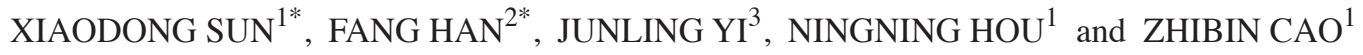 \\ Departments of ${ }^{1}$ Endocrinology and ${ }^{2}$ Pathology, The Affiliated Hospital of Weifang Medical University, \\ Weifang, Shangdong 261031; ${ }^{3}$ Department of Obstetrics, Qingdao Municipal Hospital (East), \\ Qingdao, Shandong 266003, P.R. China
}

Received October 28, 2012; Accepted February 12, 2013

DOI: $10.3892 / \mathrm{mmr} .2013 .1350$

\begin{abstract}
Serious complications as a result of type 2 diabetes mellitus (T2DM) are becoming a major health concern. In the present study, it was hypothesized that telomerase activity is upregulated in vascular smooth muscle cells (VSMCs) during proliferation in T2DM and that the application of telomerase inhibitors impedes the proliferation of VSMCs in vitro. Male Wistar rats were randomly allocated into the normal control (NC) or diabetic (DM) group. Diabetes was induced by high-fat feeding and a low dose of streptozotocin (STZ; $30 \mathrm{mg} / \mathrm{kg}$ ). Primary VSMC cultures were exposed to normal $(5.5 \mathrm{mM})$ or high $(25 \mathrm{mM})$ glucose and insulin $(100 \mathrm{nM})$ in the presence and absence of various concentrations of antisense oligoribonucleotides (ASODNs) for varying lengths of time. Telomerase activity and the proliferation of VSMCs were measured. Results showed that there was a significant increase in the levels of fasting glucose, insulin, triglycerides (TG) and free fatty acids (FFAs) in the diabetic group. Telomerase activity and the proliferation of VSMCs were significantly higher in the diabetic group in vivo and in the high glucose and insulin (HGI)-treated group in vitro $(\mathrm{P}<0.01)$. ASODNs significantly inhibited the proliferation of VSMCs in a concentration- and time-dependent manner $(\mathrm{P}<0.01)$. In conclusion, hyperglycemia and hyperinsulinemia stimulate telomerase activity and the proliferation of VSMCs, while the inhibition of telomerase activity reduces the proliferation of VSMCs, indicating that telomerase may be involved in the pathological process of diabetic vascular disease.
\end{abstract}

Correspondence to: Dr Xiaodong Sun, Department of Endocrinology, Affiliated Hospital of Weifang Medical University, No. 2428 Yuhe Road, Weifang, Shandong 261031, P.R. China

E-mail: sxdfriend@sina.com

${ }^{*}$ Contributed equally

Key words: diabetes mellitus, telomerase, vascular smooth muscle cell

\section{Introduction}

Serious complications as a result of type 2 diabetes mellitus (T2DM) are becoming a major health concern, among which atherosclerosis, the leading cause of morbidity and mortality in developed countries, is the most common disease. The most common pathological change in atherosclerosis is the proliferation of vascular smooth muscle cells (VSMCs), which occurs in response to arterial injury and plays a crucial role in the atherosclerotic process (1). Therefore, it is necessary to elucidate the molecular mechanisms of abnormal VSMC proliferation. It is known that insulin resistance, hyperinsulinemia and hyperglycemia stimulate and accelerate VSMC proliferation, hypertrophy and abnormal vascular tone in patients with T2DM (2-4). Although certain mechanisms of hyperglycemia- and hyperinsulinemia-induced VSMC proliferation have been proposed to contribute to atherosclerosis, there is limited knowledge with regard to the role of telomerase activity in VSMC proliferation (5-7).

Telomeres are DNA-protein complexes located at the ends of eukaryotic chromosomes, which maintain genome integrity and stabilize the chromosome function (8). Telomeric DNA is shortened with cell mitosis. When a critical length is reached, the telomeric ends of chromosomes lose their protective function and induce cell apoptosis. Telomerase activation increases telomere repeats on the chromosome ends, maintains genome integrity and induces cellular immortalization (9).

Studies with regard to telomerase were initially introduced in the field of oncology $(10,11)$. Recently, telomerase has also been studied in proliferative diseases, including hypertension, cardiac hypertrophy and heart failure, and is considered to be involved in the proliferation of abnormal cells and important in the pathogenesis of these diseases (12-14). Animal studies have demonstrated a relatively higher level of telomerase activation in the VSMCs of spontaneously hypertensive rats (15). Telomerase activation may induce a phenotypic change in VSMCs and play a role in cell remodeling, indicating that telomerase may be important in the regulation of tissue regeneration and cell signaling pathways in VSMC proliferation.

Since telomerase activity is associated with cell proliferation, aging and apoptosis and there is an imbalance between VSMC proliferation and apoptosis in T2DM, it was hypoth- 
esized that telomerase may also be important in VSMC proliferation in T2DM. Therefore, the aim of the present study was to investigate whether telomerase activity is upregulated in VSMC proliferation in T2DM and whether the application of telomerase inhibitors impedes VSMC proliferation in vitro. To test this hypothesis, we examined the telomerase activity of VSMCs in a rat model of type 2 diabetes and in cell cultures in vitro. We also explored the effect of telomerase inhibitors on hyperinsulinemia- and hyperglycemia-induced VSMC proliferation.

\section{Materials and methods}

Materials. Streptozotocin (STZ) and 3-(4,5-dimethylthiazol-2-yl)-2,5-diphenyl tetrazolium bromide (MTT) were purchased from Sigma (St. Louis, MO, USA). Insulin radioimmunoassay and telomerase assay kits were purchased from Linco Research, Inc. (St Louis, MO, USA) and Roche Molecular Biochemicals (Mannheim, Germany), respectively. Antisense oligoribonucleotides (ASODNs; 5'-GAGCGCGGGTCATTGTGCT-3') and sense oligoribonucleotides (SODNs; 5'-AGCACAATGACCCGCGCTC-3') were designed as telomerase inhibitors according to the rat telomerase reverse transcriptase mRNA sequence of GenBank from Beijing General Chemical Reagent Factory (Beijing, China). Additional reagents were purchased from Beijing General Chemical Reagent Factory.

Experimental animals. Six-week-old male Wistar rats (specific pathogen-free quality) were purchased from the Experimental Animal Center of Shandong University (Shandong, China) and the use of animals was approved by the Institutional Animal Care and Use Committee (IACUC; approval no. SCXK20100301). The rats were randomly allocated into the normal control (NC) or diabetes mellitus (DM) group. Rats of the NC group were fed a regular diet (8\% fat; $68 \%$ carbohydrate; $24 \%$ protein; $320 \mathrm{kcal} / 100 \mathrm{~g}$ ), whereas rats of the DM group were fed a high-fat diet $(50.10 \%$ fat, mainly saturated; $33.60 \%$ carbohydrate; $16.30 \%$ protein; $493 \mathrm{kcal} / 100 \mathrm{~g})$. After 8 weeks, rats of the DM group were administered a low dose of STZ (30 mg/ $\mathrm{kg}$ ) by intraperitoneal injection, while those of the NC group were administered the same volume of vehicle citrate buffer. Blood glucose levels were measured $72 \mathrm{~h}$ after the STZ injection. Only the rats with glucose levels $>16.7 \mathrm{mmol} / \mathrm{l}$ were considered to be a successful diabetic model (16). The diabetic model achieved was similar to type 2 diabetes in humans. Thus, the combination of a high-fat intake and low-dose STZ effectively induced type 2 diabetes by altering the expression of associated genes in major metabolic tissues (17). The rats were housed under standard laboratory conditions and had free access to water and standard rat chow. The rats were sacrificed four weeks after the successful establishment of the diabetic model.

Plasma measurements. The rats were anesthetized using pentobarbital sodium. Blood samples were then immediately collected and separated for plasma measurements. Plasma glucose was measured using the glucose oxidase method. Plasma insulin was measured using a radioimmunoassay according to the manufacturer's instructions. Plasma triglyceride (TG), cholesterol (TC) and free fatty acid (FFA) levels were measured using colorimetric assays.

Collection of VSMCs. The thoracic aorta was immediately dissected and enzymatically digested at $37^{\circ} \mathrm{C}$ for $\sim 3.5 \mathrm{~h}$ using a $0.25 \%$ trypsin solution. Following digestion, tissue fragments were explanted in a $35-\mathrm{mm}$ culture dish. Contaminated fibroblasts were separated from the VSMCs due to their differing adhesion abilities (18).

Determination of telomerase activity. Telomerase activity in VSMCs was examined using the telomeric repeat amplification protocol enzyme-linked immunosorbent assay (TRAP-ELISA), according to the manufacturer's instructions (19). Briefly, the same amount of protein from each sample was incubated with a biotin-labeled telomerase substrate primer at $25^{\circ} \mathrm{C}$ for $15 \mathrm{~min}$ and primer extension was carried out using polymerase chain reaction (PCR; 30 cycles of $94^{\circ} \mathrm{C}$ for $30 \mathrm{sec}, 60^{\circ} \mathrm{C}$ for $30 \mathrm{sec}$ and $72^{\circ} \mathrm{C}$ for $90 \mathrm{sec}$ ). The PCR-amplified products were hybridized for $120 \mathrm{~min}$ and incubated with a peroxidase-labeled anti-digoxigenin antibody for $30 \mathrm{~min}$. Following the addition of peroxidase substrate, the optical density (OD) of each sample was measured at $450 \mathrm{~nm}$ with a reference wavelength of $690 \mathrm{~nm}$ using an enzyme immunoassay microplate reader. The OD values were reported as A450-A690 nm. Samples were considered positive when the OD values were $\geq 0.2$.

VSMC proliferation assay. VSMC proliferation was measured using the MTT assay (20). Briefly, VSMCs were harvested by trypsinization and plated in a 96-well plate at a density of $1 \times 10^{5}$ cells $/ \mathrm{ml}$. VSMCs were then grown in $100 \mu \mathrm{l}$ of medium at $37^{\circ} \mathrm{C}$ for $24 \mathrm{~h}$, followed by incubation with $20 \mu \mathrm{l} \mathrm{MTT}$ for $4 \mathrm{~h}$. Then, $150 \mu \mathrm{l}$ dimethyl sulfoxide (DMSO) was added to each well and the absorbance was measured at a wavelength of $490 \mathrm{~nm}$ using a microplate reader.

In vitro experiment. VSMCs from six-week-old normal Wistar rats were cultured using the tissue explants technique and were identified using an immunofluorescence staining method (21). The 3-5 generations of cells used in all experiments were allocated into four groups: the normal control, high glucose and insulin (HGI), HGI + low concentration of ASODN or SODN and HGI + high concentration of ASODN or SODN groups. After the cells were serum starved for $24 \mathrm{~h}$, they were exposed to normal glucose $(5.5 \mathrm{mM})$ or high glucose $(25 \mathrm{mM})$ and insulin $(100 \mathrm{nM})$ in the presence and absence of ASODN or SODN for $24 \mathrm{~h}$ and then subjected to an assessment of telomerase activity and cell proliferation using the methods mentioned previously. In certain experiments, $19.5 \mathrm{mM}$ of mannitol was used to control variations in osmotic pressure.

Statistical analysis. Data were subjected to statistical analysis using the SPSS 13.0 statistical package. The data were expressed as the mean \pm standard deviation (SD). The statistical tests used were Student's t-test or one way ANOVA. The number of inter-group differences were detected using the Student-Newman-Keuls (SNK) method. The association between two variables was analyzed using Pearson's 
Table I. Serum levels of fasting glucose, insulin, FFAs and TG in the studied groups $(\mathrm{n}=10)$.

\begin{tabular}{lcccc}
\hline Group & $\begin{array}{c}\text { Glucose } \\
(\mathrm{mmol} / \mathrm{l})\end{array}$ & $\begin{array}{c}\text { Insulin } \\
(\mu \mathrm{IU} / \mathrm{ml})\end{array}$ & $\begin{array}{c}\text { FFAs } \\
(\mu \mathrm{mol} / \mathrm{l})\end{array}$ & $\begin{array}{c}\text { TGs } \\
(\mathrm{mmol} / \mathrm{l})\end{array}$ \\
\hline $\mathrm{NC}$ & $5.88 \pm 0.73$ & $11.24 \pm 0.68$ & $301.63 \pm 78.29$ & $0.80 \pm 0.32$ \\
$\mathrm{DM}$ & $15.82 \pm 3.21^{\mathrm{a}}$ & $18.99 \pm 3.68^{\mathrm{a}}$ & $934.35 \pm 93.39^{\mathrm{a}}$ & $1.89 \pm 0.33^{\mathrm{a}}$ \\
\hline
\end{tabular}

${ }^{\text {aP }}<0.01$ compared with the control group. Data are shown as the mean \pm SD. FFAs, free fatty acids; TGs, triglycerides; NC, normal control; DM, diabetes mellitus; SD, standard deviation.

Table II. Telomerase activity of VSMCs in the in vitro groups.

\begin{tabular}{lll}
\hline Group & \multicolumn{1}{c}{$24 \mathrm{~h}$} & \multicolumn{1}{c}{$72 \mathrm{~h}$} \\
\hline NC & $0.105 \pm 0.055$ & $0.125 \pm 0.033$ \\
HGI & $0.599 \pm 0.079^{\mathrm{a}}$ & $0.756 \pm 0.053^{\mathrm{a}}$ \\
SODN $(5 \mu \mathrm{m})$ & $0.572 \pm 0.023^{\mathrm{a}}$ & $0.731 \pm 0.083^{\mathrm{a}}$ \\
ASODN $(5 \mu \mathrm{m})$ & $0.425 \pm 0.086^{\mathrm{a}, \mathrm{b}}$ & $0.535 \pm 0.053^{\mathrm{a}, \mathrm{b}}$ \\
SODN $(10 \mu \mathrm{m})$ & $0.562 \pm 0.026^{\mathrm{a}}$ & $0.709 \pm 0.094^{\mathrm{a}}$ \\
ASODN $(10 \mu \mathrm{m})$ & $0.365 \pm 0.060^{\mathrm{a}, \mathrm{b}}$ & $0.406 \pm 0.069^{\mathrm{a}, \mathrm{b}}$ \\
SODN $(20 \mu \mathrm{m})$ & $0.559 \pm 0.035^{\mathrm{a}}$ & $0.692 \pm 0.066^{\mathrm{a}}$ \\
ASODN $(20 \mu \mathrm{m})$ & $0.174 \pm 0.039^{\mathrm{a}, \mathrm{b}}$ & $0.185 \pm 0.046^{\mathrm{a}, \mathrm{b}}$ \\
Negative control & $0.105 \pm 0.023$ & \\
Positive control & $2.256 \pm 0.858^{\mathrm{a}}$ & \\
\hline
\end{tabular}

${ }^{\mathrm{a}} \mathrm{P}<0.01$ compared with the $\mathrm{NC}$ group; ${ }^{\text {b }} \mathrm{P}<0.01$ compared with the HGI group. Data are shown as the mean \pm SD. VSMCs, vascular smooth muscle cells; NC, normal control; HGI, high glucose and insulin; SODN, sense oligoribonucleotides; ASODN, antisense oligoribonucleotides; SD, standard deviation.

correlation. $\mathrm{P}<0.05$ was considered to indicate a statistically significant difference.

\section{Results}

Blood parameters of rats in the studied groups. In the DM group, a significant increase was observed in the levels of fasting glucose $(15.82 \pm 3.21$ vs. $5.88 \pm 0.73 \mathrm{mmol} / \mathrm{l}, \mathrm{P}<0.01)$, insulin $(18.99 \pm 3.68$ vs. $11.24 \pm 0.68 \mu \mathrm{IU} / \mathrm{ml}, \mathrm{P}<0.01)$, FFAs $(934.35 \pm 93.39$ vs. $301.63 \pm 78.29 \mu \mathrm{mol} / 1, \mathrm{P}<0.01)$ and TGs $(1.89 \pm 0.33$ vs. $0.80 \pm 0.32 \mathrm{mmol} / \mathrm{l}, \mathrm{P}<0.01$; Table I).

Telomerase activity and proliferation of VSMCs. The expression of telomerase activity and the degree of VSMC proliferation were significantly higher in the DM compared with the normal control group ( $\mathrm{P}<0.01$; Fig. 1). To determine whether the changes in telomerase activity and proliferation of VSMCs were induced by HGI, we investigated the effect of HGI treatment on VSMCs in vitro. The results showed that telomerase activation and the proliferation of VSMCs were higher when induced by HGI. Since the addition of $19.5 \mathrm{mM}$ of mannitol caused no significant changes, we were able to
A

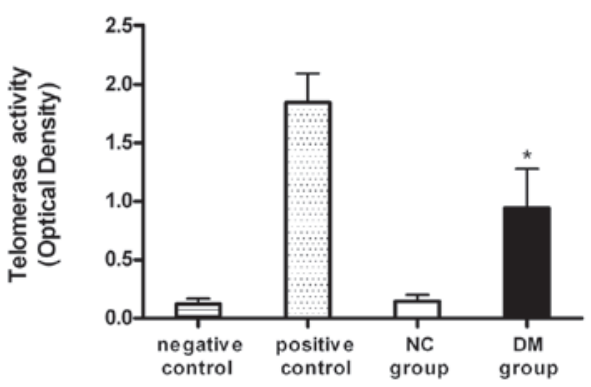

B

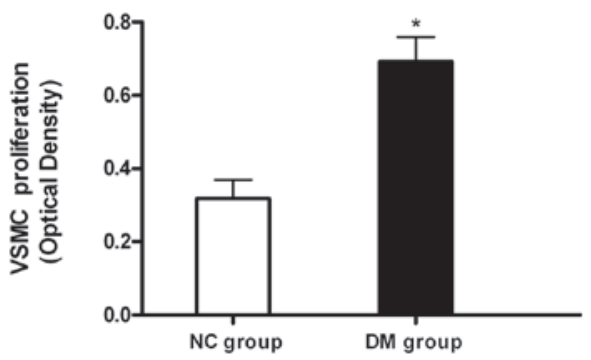

Figure 1. (A) Telomerase activity and (B) the proliferation of VSMCs in the normal control (NC) and diabetes mellitus (DM) groups. ${ }^{*} \mathrm{P}<0.01$ vs. the NC group. VSMCs, vascular smooth muscle cells.

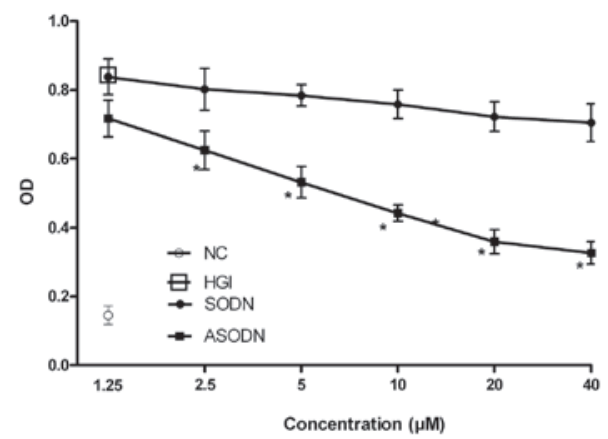

Figure 2. Proliferation of VSMCs induced by various concentrations of oligoribonucleotides. ${ }^{*} \mathrm{P}<0.01$ vs. the HGI group. OD, optical density; NC, normal control; HGI, high glucose and insulin; SODN, sense oligonucleotides; ASODN, antisense oligoribonucleotides; VSMCs, vascular smooth muscle cells.

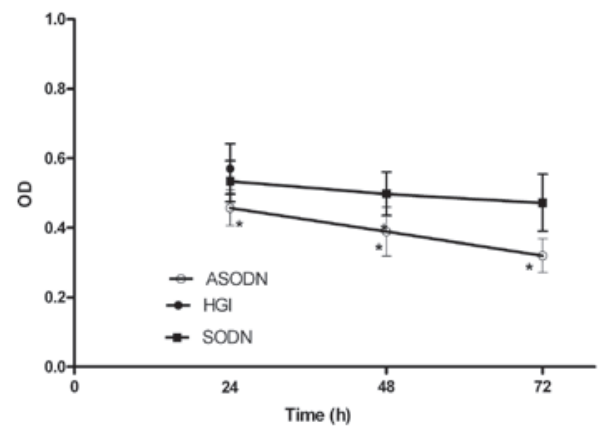

Figure 3. Proliferation of VSMCs induced by $20 \mu \mathrm{M}$ antisense oligoribonucleotides (ASODN) at 24, 48 and $72 \mathrm{~h}$. ${ }^{*} \mathrm{P}<0.01$ vs. the HGI group. OD, optical density; HGI high glucose and insulin; SODN; sense oligonucleotides; VSMCs, vascular smooth muscle cells.

determine that the glucose effect was not a result of hyperosmolarity. After the intervention of ASODN, telomerase activity decreased in a concentration-dependent manner 
$(\mathrm{P}<0.05)$. However, the same concentration of SODN did not significantly inhibit telomerase activity (Table II).

Various concentrations of oligoribonucleotides induce VSMC proliferation. With regard to the in vitro experiments, HGI significantly promoted VSMC proliferation. In order to examine the role of telomerase activity in VSMC proliferation, a telomerase inhibitor (ASODN) was used to inhibit the proliferation of VSMCs in vitro. The results showed that ASODN significantly inhibited the proliferation of VSMCs in a concentration-dependent manner $(\mathrm{P}<0.05$; Fig. 2). Additionally, the treatment of VSMCs induced by HGI with $20 \mu \mathrm{M}$ ASODN significantly inhibited the proliferation of VSMCs. At 24, 48 and $72 \mathrm{~h}$ after treatment, the inhibition rates were 19.8, 31.8 and $43.9 \%$, respectively, indicating a time-dependent inhibition of VSMC proliferation. SODN also inhibited the proliferation of VSMCs. However, SODN caused no significant effect on VSMC proliferation compared with ASODN (Fig. 3).

\section{Discussion}

In the present study, evidence that telomerase activity and the proliferation of VSMCs are increased in the rat model of T2DM and that HGI causes increased telomerase activation and proliferation of VSMCs in vitro is provided. It was further demonstrated that VSMC proliferation was significantly reduced by the intervention of telomerase inhibitors (ASODNs) in vitro.

T2DM is characterized by target-tissue resistance to insulin, which often causes hyperglycemia and hyperinsulinemia. In the present study, STZ-induced T2DM rats showed a significant increase in fasting plasma glucose and insulin levels compared with normal rats, indicating a successful model of T2DM. This diabetic model was similar to T2DM in humans, as the high-fat diet initiated a state of insulin resistance and then the treatment with a low dose of STZ established a relative insulin deficiency (22). It is known that hyperglycemia and hyperinsulinemia are associated with atherosclerosis in T2DM and this is mainly caused by the abnormal proliferation of VSMCs. Under physiological conditions, there is a balance between the proliferation and apoptosis of VSMCs. When this balance is disturbed by hyperglycemia and hyperinsulinemia, VSMC proliferation is increased and promotes the development of atherosclerosis (23).

Telomerase activity is known to be closely associated with the cell proliferation and apoptosis of VSMCs. Telomerase is wrapped in the cell nucleus and is released to repair chromosomal DNA when cell division or DNA damage has occurred. Thus, telomerase activity is inhibited in normal cells and is activated in tumor or proliferating cells in normal tissues. Telomerase is a marker of cell proliferation at the molecular level and telomerase activity is expressed at various levels in tissues with hyperplasia (24). Additionally, previous studies have demonstrated that telomerase activation may induce phenotypic changes in VSMCs by being involved in cell remodeling processes and that it is important in the signaling pathway of VSMC proliferation $(15,25)$. There are different levels of telomerase upregulation in the abnormal proliferation of VSMCs. In the present study, telomerase activity and the degree of VSMC proliferation were significantly higher in diabetic compared with control rats. This finding indicates that factors, including lipids, viral infection and hypoxia, lead to VSMC proliferation, which initiates the development of atherosclerotic plaques. To determine whether hyperglycemia and hyperinsulinemia induce upregulated telomerase activity, the telomerase activity in HGI-induced VSMC proliferation was determined. The results showed that the expression of telomerase activity was higher in HGI-induced VSMCs compared with the normal control group, suggesting that telomerase activity is upregulated in hyperglycemia- and hyperinsulinemia-induced VSMC proliferation in T2DM.

Telomerase is a regulating enzyme. The telomerase reverse transcriptase (TERT) catalytic subunit is the key component that regulates telomerase activity. The TERT expression level is closely associated with telomerase activity. Thus, ASODNs, the synthetic short-chain nucleic acids which bind to and degrade TERT mRNA, have been considered ideal for the inhibition of telomerase activity (26). In the present study, ASODN significantly inhibited VSMC proliferation in a dose- and time-dependent manner. Although SODN demonstrated an inhibitory effect, no significant difference was identified when compared with the HGI group, indicating that this inhibitory effect is caused by a specific base sequence.

In conclusion, the in vivo and in vitro experiments in this study have shown that hyperglycemia and hyperinsulinemia stimulated the telomerase activity and proliferation of VSMCs, while the inhibition of telomerase activity reduced the proliferation of VSMCs, indicating that telomerase may be involved in the pathological process of diabetic vascular disease. The specific molecular mechanisms require further investigation to be fully elucidated. Telomerase inhibition may provide a unique approach for the treatment of vascular complications in T2DM.

\section{References}

1. Bruemmer D and Law RE: Thiazolidinedione regulation of smooth muscle cell proliferation. Am J Med 115: 87-92, 2003.

2. Carmody BJ, Arora S, Wakefield MC, Weber M, Fox CJ and Sidawy AN: Progesterone inhibits human infragenicular arterial smooth muscle cell proliferation induced by high glucose and insulin concentrations. J Vasc Surg 36: 833-838, 2002.

3. Cifarelli V, Luppi P, Tse HM, He J, Piganelli J and Trucco M: Human proinsulin C-peptide reduces high glucose-induced proliferation and NF- $\mathrm{BB}$ activation in vascular smooth muscle cells. Atherosclerosis 201: 248-257, 2008

4. Isenović ER, Kedees MH, Tepavcević S, Milosavljević T, Korićanac G, Trpković A and Marche P: Role of PI3K/AKT, cPLA2 and ERK1/2 signaling pathways in insulin regulation of vascular smooth muscle cells proliferation. Cardiovasc Hematol Disord Drug Targets 9: 172-180, 2009.

5. Gizard F, Nomiyama T, Zhao Y, et al: The PPARalpha/p16INK4a pathway inhibits vascular smooth muscle cell proliferation by repressing cell cycle-dependent telomerase activation. Circ Res 103: 1155-1163, 2008.

6. Herbert KE, Mistry Y, Hastings R, Poolman T, Niklason L and Williams B: Angiotensin II-mediated oxidative DNA damage accelerates cellular senescence in cultured human vascular smooth muscle cells via telomere-dependent and independent pathways. Circ Res 102: 201-208, 2008.

7. Jacob T, Hingorani A and Ascher E: Evidence for telomerase activation in VSMCs exposed to hyperglycemic and hyperhomocysteinemic conditions. Angiology 60: 562-568, 2009.

8. Serrano AL and Andrés V: Telomeres and cardiovascular disease: does size matter? Circ Res 94: 575-584, 2004.

9. Gancarcíková M, Zemanová Z, Brezinová J, Berková A, Vcelíková S, Smigová J and Michalová K: The role of telomeres and telomerase complex in haematological neoplasia: the length of telomeres as a marker of carcinogenesis and prognosis of disease. Prague Med Rep 111: 91-105, 2010. 
10. Jones CH, Pepper C and Baird DM: Telomere dysfunction and its role in haematological cancer. Br J Haematol 156: 573-587, 2012.

11. Sprouse AA, Steding CE and Herbert BS: Pharmaceutical regulation of telomerase and its clinical potential. J Cell Mol Med 16: $1-7,2012$.

12. Cesselli D, Beltrami AP, D'Aurizio F, et al: Effects of age and heart failure on human cardiac stem cell function. Am J Pathol 179: 349-366, 2011.

13. El Assar M, Angulo J, Vallejo S, Peiró C, Sánchez-Ferrer CF and Rodríguez-Mañas L: Mechanisms involved in the aging-induced vascular dysfunction. Front Physiol 3: 132, 2012.

14. Fyhrquist $F$ and Saijonmaa O: Telomere length and cardiovascular aging. Ann Med 44 (Suppl 1): S138-S142, 2012.

15. Cao Y, Li H, Mu FT, Ebisui O, Funder JW and Liu JP: Telomerase activation causes vascular smooth muscle cell proliferation in genetic hypertension. FASEB J 16: 96-98, 2002.

16. Coppey LJ, Davidson EP, Rinehart TW, Gellett JS, Oltman CL, Lund DD and Yorek MA: ACE inhibitor or angiotensin II receptor antagonist attenuates diabetic neuropathy in streptozotocin-induced diabetic rats. Diabetes 55: 341-348, 2006.

17. Wang HJ, Jin YX, Shen W, Neng J, Wu T, Li YJ and Fu ZW: Low dose streptozotocin (STZ) combined with high energy intake can effectively induce type 2 diabetes through altering the related gene expression. Asia Pac J Clin Nutr 16 (Suppl 1): 412-417, 2007.

18. Wang Y, Qiao L, Qiu J, Mi W, Han Y and Zhong C: Establishing primary cultures of vascular smooth muscle cells from the spira modiolar artery. Int J Pediatr Otorhinolaryngol 76: 1082-1086, 2012.

19. He X, Qiao Q, Ge N, et al: Irradiation-induced telomerase activity and gastric cancer risk: a case-control analysis in a Chinese Han population. BMC Cancer 10: 312, 2010.
20. Huang J, Li LS, Yang DL, Gong QH, Deng J and Huang XN: Inhibitory effect of ginsenoside $\mathrm{rg} 1$ on vascular smooth muscle cell proliferation induced by PDGF-BB is involved in nitric oxide formation. Evid Based Complement Alternat Med 2012: 314395, 2012.

21. McMurray HF, Parrott DP and Bowyer DE: A standardised method of culturing aortic explants, suitable for the study of factors affecting the phenotypic modulation, migration and proliferation of aortic smooth muscle cells. Atherosclerosis 86 : 227-237, 1991.

22. Sun X, Han F, Yi J, Han L and Wang B: Effect of aspirin on the expression of hepatocyte NF-kappaB and serum TNF-alpha in streptozotocin-induced type 2 diabetic rats. J Korean Med Sci 26: 765-770, 2011.

23. Jacob T, Clouden N, Hingorani A and Ascher E: The effect of cotinine on telomerase activity in human vascular smooth muscle cells. J Cardiovasc Surg (Torino) 50: 345-349, 2009.

24. Belair CD, Yeager TR, Lopez PM and Reznikoff CA: Telomerase activity: a biomarker of cell proliferation, not malignant transformation. Proc Natl Acad Sci USA 94: 13677-13682, 1997.

25. Matthews C, Gorenne I, Scott S, et al: Vascular smooth muscle cells undergo telomere-based senescence in human atherosclerosis: effects of telomerase and oxidative stress. Circ Res 99: 156-164, 2006.

26. Chen H, Li Y and Tollefsbol TO: Strategies targeting telomerase inhibition. Mol Biotechnol 41: 194-199, 2009. 